\title{
On propagation of high-energy photon in a medium in presence of an external field
}

\author{
V. N. Baier and V. M. Katkov \\ Budker Institute of Nuclear Physics, \\ Novosibirsk, 630090, Russia
}

December 23, 2018

\begin{abstract}
The polarization tensor is calculated which originates from interaction of a photon with the electron-positron field. The effects of multiple scattering of electrons and positrons in a medium side by side with external fields is included. The corresponding general representation of polarization tensor is found in the form of two-dimensional integral. The both effects may be essential for propagation of high-energy photons in oriented single crystals.
\end{abstract}




\section{Introduction}

As known, the propagation of electromagnetic wave in a medium is defined by its dielectric tensor $\mathcal{E}_{i k}(\omega)$. For relatively low frequency $\omega$ (e.g. visible light) the dielectric tensor is defined by atomic phenomena. When the frequency of wave is much higher the atomic frequencies, the dielectric tensor has a form

$$
\mathcal{E}_{i k}(\omega)=\delta_{i k} \mathcal{E}(\omega), \quad \mathcal{E}(\omega)=1-\frac{\omega_{0}^{2}}{\omega^{2}}, \quad \omega_{0}^{2}=\frac{4 \pi n_{e} e^{2}}{m}
$$

where $n_{e}$ is the number density of electrons in a medium, $m(e)$ is the electron mass (charge), $\omega_{0}$ is the plasma frequency, in any medium $\omega_{0}<100 \mathrm{eV}$. So that for $\omega \gg \omega_{0}$ an influence of atomic phenomena on propagation of electromagnetic wave in a medium becomes small. It should be noted that for radiation of highenergy particles the characteristic frequency is $\gamma \omega_{0}, \gamma$ is the Lorentz factor.

At very high energy the nonlinear effects of QED enter into game. These effects are due to interaction of photon with the electron-positron field. One of them is the polarization of the vacuum by a photon. In the presence of an external electromagnetic field the polarization of vacuum was considered first in the pioneer papers [1]. In the strong field this effect can be essential for propagation of high-energy photons [2], [3].

To evaluate the polarization tensor one has to consider the amplitude of photon scattering which included the polarization operator. We use the quasiclassical operator method [4], [3], [5], 6]. In this method the mentioned amplitude is described by diagram where the virtual electron-positron pair is first created by the initial photon with 4-momentum $k(\omega, \mathbf{k})$ and polarization $\mathbf{e}_{1}$ and then annihilate into final photon with 4-momentum $k$ and polarization $\mathbf{e}_{2}$. This corresponds to use of the non-covariant perturbation theory where at high energies $(\omega \gg m)$ the contribution of this diagram survives only. For this energy of photon this process occurs in a rather long time (or at a rather long distance) known as the lifetime of the virtual state

$$
l_{f}=\frac{\omega}{2 q_{c}^{2}},
$$

where $q_{c} \geq m$ is the characteristic transverse momentum of the process, the system $\hbar=c=1$ is used. When the virtual electron (or positron) is moving in a medium it scatters on atoms and changes the velocity under influence of external electromagnetic field. The mean square of momentum transfer to the electron from a medium and an external field on the distance $l_{f}$ is

$$
\begin{aligned}
& q_{f}^{2}=q_{s}^{2}+\mathbf{q}_{F}^{2}, \quad q_{s}^{2}=4 \pi Z^{2} \alpha^{2} n_{a} L l_{f}, \quad L \equiv L\left(q_{c}^{2}\right)=\ln \left(q_{c}^{2} a^{2}\right), \\
& \mathbf{q}_{F}=e \mathbf{F}_{e} l_{f}, \quad \mathbf{F}_{e}=\mathbf{E}_{\perp}+\mathbf{v} \times \mathbf{H}
\end{aligned}
$$

where $\alpha=e^{2}=1 / 137, Z$ is the charge of nucleus, $n_{a}$ is the number density of atoms in the medium, $a$ is the screening radius of atom, $\mathbf{E}_{\perp}$ is the electric field strength transverse to the velocity of particle $\mathbf{v} \simeq \mathbf{n}=\mathbf{k} / \omega, \mathbf{H}$ is the magnetic field strength. 
In the case of small momentum transfer $q_{f} \equiv \sqrt{q_{f}^{2}} \ll m$ the influence of a medium and an external field is weak, in this case $q_{c}=m$. At high energy it is possible that $q_{c} \geq m$. In this case the characteristic value of the momentum transfer (giving the main contribution into the spectral probability) is defined by the value of $q_{f}$. The self-consistency condition is

$$
\begin{aligned}
& q_{c}^{2}=q_{f}^{2}=\frac{2 \pi \omega Z^{2} \alpha^{2} n_{a} L\left(q_{f}\right)}{q_{f}^{2}}+\frac{m^{6} \boldsymbol{\kappa}^{2}}{4 q_{f}^{4}} \geq m^{2}, \boldsymbol{\kappa}=\frac{\omega}{m^{3}} e \mathbf{F}, \\
& \mathbf{F}=\mathbf{E}-\mathbf{n}(\mathbf{n E})+\mathbf{n} \times \mathbf{H},
\end{aligned}
$$

here $\kappa=|\boldsymbol{\kappa}|$ is known parameter characterising the pair production process in a homogeneous external field $\mathbf{F}$. With $q_{c}$ increase the lifetime of the virtual state (1.2) decreases.

We will use the following normalization condition for the amplitude under consideration

$$
M=2 \omega \Delta \omega .
$$

The amplitude $M$ is the contraction of the tensor $e_{j}^{(i) *} e_{k}^{(f)}\left(\mathbf{e}^{(i)}\right.$ and $\mathbf{e}^{(f)}$ are the polarization vectors of the initial and the final photons) and the polarization tensor $M_{j k}$. We select the basic vectors as

$$
\mathbf{e}_{1}=\frac{\mathbf{F}}{|\mathbf{F}|}, \quad \mathbf{e}_{2}=\mathbf{n} \times \mathbf{e}_{1}
$$

Since the tensor $M_{j k}$ is invariant under the space inversion then in the selected basic vectors it has the diagonal form

$$
M_{j k}=\frac{1}{2}\left[\delta_{j k}\left(M_{11}+M_{22}\right)+\left(e_{1 j} e_{1 k}-e_{2 j} e_{2 k}\right)\left(M_{11}-M_{22}\right)\right]
$$

In absence of external field it is convenient to describe the process of photon scattering using the helicity polarization vector $\mathbf{e}_{\lambda}(\lambda= \pm 1)$ connected with momentum transfer $\boldsymbol{\Delta}$ (see Eq.(2.34) in [9]). In presence of external field and for $\boldsymbol{\Delta}=0$ we choose the polarization vectors in the following way:

$$
\mathbf{e}_{\lambda}=\frac{1}{\sqrt{2}}\left(\mathbf{e}_{1}+i \lambda \mathbf{e}_{2}\right), \quad\left(\mathbf{e}_{\lambda} \mathbf{e}_{\lambda}^{*}\right)=1, \quad\left(\mathbf{e}_{\lambda} \mathbf{e}_{-\lambda}^{*}\right)=0, \quad \mathbf{e}_{\lambda} \times \mathbf{n}=i \lambda \mathbf{e}_{\lambda} .
$$

In terms of helicity amplitudes $M_{++}$and $M_{+-}$the tensor $M_{j k}$ and the corresponding dielectric tensor $\mathcal{E}_{j k}$ has a form

$$
\begin{aligned}
& M_{j k}=\delta_{j k} k_{++}^{2}+\left(e_{1 j} e_{1 k}-e_{2 j} e_{2 k}\right) k_{+-}^{2}, \\
& \mathcal{E}_{j k}=\delta_{j k}-\frac{1}{\omega^{2}} M_{j k}, \quad k_{++}^{2} \equiv M_{++}, \quad k_{+-}^{2} \equiv M_{+-}
\end{aligned}
$$

The polarization tensor $k_{j k}^{2}$ is diagonal in the basic vectors $\mathbf{e}_{1}$ and $\mathbf{e}_{2}$ (1.6). The corresponding mass squared are

$$
k_{1}^{2} \equiv k_{11}^{2}=k_{++}^{2}+k_{+-}^{2}, \quad k_{2}^{2} \equiv k_{22}^{2}=k_{++}^{2}-k_{+-}^{2}
$$


The probability of pair creation by a photon with polarization $\mathbf{e}$ is

$$
W_{p}^{F}(\mathbf{e})=-\frac{1}{\omega} \operatorname{Im}\left[\left(\mathbf{e e}_{1}\right)^{2} k_{1}^{2}+\left(\mathbf{e e}_{2}\right)^{2} k_{2}^{2}\right]=-\frac{1}{\omega} \operatorname{Im}\left[k_{++}^{2}+\xi_{3} k_{+-}^{2}\right],
$$

where $\xi_{3}$ is the Stokes' parameter. For unpolarized photon one has

$$
W_{p}^{F}=-\frac{1}{\omega} \operatorname{Im} k_{++}^{2}
$$

\section{Polarization tensor}

The polarization of a medium by a high-energy photon in the presence of an external field is described by the amplitudes of photon forward scattering. The general representation of photon scattering amplitudes without change of helicity $M_{++}=M_{--}$and with helicity flip $M_{+-}=M_{-+}$was obtained in 9 (see Eq.(3.14)). In the mentioned paper [9] the helicity amplitudes were normalized by the condition $\operatorname{Im} M_{++}=\omega \sigma_{p}(\omega)$, where $\sigma_{p}(\omega)$ is the total cross section of a production of electron-positron pair by a photon with energy $\omega$. In the case under consideration we are interested in corrections to the energy (mass) of photon and it is convenient to use the normalization (1.11) and (1.12)

$$
k_{\lambda \lambda^{\prime}}^{2}=-2 \alpha m^{2} \int_{0}^{\omega} T_{\lambda \lambda^{\prime}} \frac{\omega d \varepsilon}{\varepsilon \varepsilon^{\prime}},
$$

where

$$
\begin{aligned}
& T_{++}=T_{--}, \quad T_{+-}=T_{-+}, \\
& T_{++}=\left\langle 0\left|s_{1}\left(G^{-1}-G_{0}^{-1}\right)+s_{2} \mathbf{p}\left(G^{-1}-G_{0}^{-1}\right) \mathbf{p}\right| 0\right\rangle \\
& T_{+-}=-2\left\langle 0\left|s_{3}\left(\mathbf{e}_{-}^{*} \mathbf{p}\right)\left(G^{-1}-G_{0}^{-1}\right)\left(\mathbf{e}_{+} \mathbf{p}\right)\right| 0\right\rangle, s_{1}=1, s_{2}=\frac{\varepsilon^{2}+\varepsilon^{\prime 2}}{\omega^{2}}, \\
& s_{3}=\frac{2 \varepsilon \varepsilon^{\prime}}{\omega^{2}}, \quad G=\mathcal{H}+1, \mathcal{H}=\mathbf{p}^{2}+V_{p}(\varrho), G_{0}=\mathbf{p}^{2}+1, \quad \varepsilon^{\prime}=\omega-\varepsilon .
\end{aligned}
$$

These expressions are similar to the expressions for probabilities of bremsstrahlung [7] and pair creation by photon [8] when the involved charged particles are subjected to the multiple scattering in a medium. The developed approach is given in these papers in detail. The same method was used in [9] for analysis of coherent scattering of a photon in a medium. In absence of an external field one can use the results of this paper putting the momentum transfer $\boldsymbol{\Delta}=0$. A modification of radiative correction under simultaneous influence of multiple scattering

and homogeneous electromagnetic field was considered recently by authors in [10], where the anomalous magnetic moment of an electron was considered. The potential $V_{p}(\varrho)$ can be obtained from Eqs.(2.7)-(2.11) of [10 with the help of standard substitutions $\omega \rightarrow-\omega, \varepsilon \rightarrow-\varepsilon$ :

$$
V_{p}(\boldsymbol{\varrho})=-i V(\varrho)+2 \boldsymbol{\kappa}^{\prime} \varrho, \quad \boldsymbol{\kappa}^{\prime}=\frac{\varepsilon \varepsilon^{\prime}}{\omega^{2}} \boldsymbol{\kappa}
$$


where $\boldsymbol{\kappa}$ and $\mathbf{F}$ are defined in Eq.(1.4). The potential $V(\varrho)$ has the form [7], [8]:

$$
\begin{aligned}
& V(\varrho)=Q \varrho^{2}\left(L_{1}+\ln \frac{4}{\varrho^{2}}-2 C\right), \quad Q=\frac{2 \pi Z^{2} \alpha^{2} \varepsilon \varepsilon^{\prime} n_{a}}{m^{4} \omega}, \quad L_{1}=\ln \frac{a_{s 2}^{2}}{\lambda_{c}^{2}}, \\
& \frac{a_{s 2}}{\lambda_{c}}=183 Z^{-1 / 3} \mathrm{e}^{-f}, \quad f=f(Z \alpha)=(Z \alpha)^{2} \sum_{k=1}^{\infty} \frac{1}{k\left(k^{2}+(Z \alpha)^{2}\right)},
\end{aligned}
$$

here $C=0.577216 \ldots$ is Euler's constant, $n_{a}$ is defined in (1.3), $\varrho$ is the coordinate in the two-dimensional space measured in the Compton wavelength $\lambda_{c}$, which is conjugate to the space of the transverse momentum transfers measured in the electron mass $m$.

We split the potential $V_{p}(\varrho)$ in the same way as in Eqs.(2.9)-(2.11) of [10]:

$$
\begin{aligned}
& V_{p}(\boldsymbol{\varrho})=V_{p F}(\boldsymbol{\varrho})-i v(\varrho), \quad V_{p F}(\boldsymbol{\varrho})=-i V_{c}(\boldsymbol{\varrho})+2 \boldsymbol{\kappa}^{\prime} \boldsymbol{\varrho}, \quad V_{c}(\boldsymbol{\varrho})=q \boldsymbol{\varrho}^{2}, \\
& q=Q L_{c} \quad, L_{c} \equiv L\left(\varrho_{c}\right)=\ln \frac{a_{s 2}^{2}}{\lambda_{c}^{2} \varrho_{c}^{2}}, \quad v(\varrho)=-\frac{q \boldsymbol{\varrho}^{2}}{L_{c}}\left(\ln \frac{\boldsymbol{\varrho}^{2}}{4 \varrho_{c}^{2}}+2 C\right) .
\end{aligned}
$$

Here the parameter $\varrho_{c}$ is defined by the set of equations (compare with Eq.(2.20) of [10]):

$$
\begin{aligned}
& \varrho_{c}=1 \text { for } 4\left(\boldsymbol{\kappa}^{\prime 2}+Q L_{1}\right) \leq 1 \\
& 4 \varrho_{c}^{4}\left(\boldsymbol{\kappa}^{\prime 2} \varrho_{c}^{2}+Q L_{c}\right)=1 \text { for } 4\left(\boldsymbol{\kappa}^{\prime 2}+Q L_{1}\right) \geq 1,
\end{aligned}
$$

According to this splitting and taking into account the addition to the potential (2.3) we present the propagators in Eq.(2.2) as

$$
G^{-1}-G_{0}^{-1}=G^{-1}-G_{p F}^{-1}+G_{p F}^{-1}-G_{0}^{-1},
$$

where

$$
G_{p F}=\mathcal{H}_{p F}+1, \quad G=G_{p F}-i v, \quad \mathcal{H}_{p F}=\mathbf{p}^{2}+V_{p F} .
$$

The representation of the propagator $G^{-1}$ permits to carry out its decomposition over the "perturbation" $v$

$$
G^{-1}-G_{p F}^{-1}=G_{p F}^{-1} i v G_{p F}^{-1}+G_{p F}^{-1} i v G_{p F}^{-1} i v G_{p F}^{-1}+\ldots
$$

The procedure of matrix elements calculation in this decomposition was formulated in [7], see Eqs.(2.30), (2.31). Here the basic matrix element is $<\varrho_{1}\left|G_{p F}^{-1}\right| \varrho_{2}>$. This matrix element can be obtained from Eq.(2.15) in [10] making the substitutions: $\omega \rightarrow-\omega, \varepsilon \rightarrow-\varepsilon, \chi / u \rightarrow \boldsymbol{\kappa}^{\prime}$

$$
\begin{aligned}
& <\varrho_{1}\left|G_{p F}^{-1}\right| \varrho_{2}>=i \int_{0}^{\infty} d t \exp (-i t) K_{p F}\left(\varrho_{1}, \varrho_{2}, t\right), \\
& K_{p F}\left(\varrho_{1}, \varrho_{2}, t\right)=K_{c}\left(\varrho_{1}, \varrho_{2}, t\right) K_{\kappa}\left(\varrho_{1}, \varrho_{2}, t\right),
\end{aligned}
$$

where

$$
\begin{aligned}
& K_{c}\left(\varrho_{1}, \varrho_{2}, t\right)=\frac{\nu}{4 \pi i \sinh \nu t} \exp \left\{\frac{i \nu}{4}\left[\left(\varrho_{1}^{2}+\boldsymbol{\varrho}_{2}^{2}\right) \operatorname{coth} \nu t-\frac{2}{\sinh \nu t} \boldsymbol{\varrho}_{1} \varrho_{2}\right]\right\} \\
& K_{\kappa}\left(\varrho_{1}, \varrho_{2}, t\right)=\exp \left[-\frac{4 i \boldsymbol{\kappa}^{\prime 2} t}{\nu^{2}}\left(1-\frac{2}{\nu t} \tanh \frac{\nu t}{2}\right)-\frac{2 i}{\nu} \boldsymbol{\kappa}^{\prime}\left(\varrho_{1}+\varrho_{2}\right) \tanh \frac{\nu t}{2}\right] .
\end{aligned}
$$


where $\nu=2 \sqrt{i q}, q$ is defined in (2.5).

In the present paper we restrict ourselves to the main term in the decomposition (2.7). This means that result will have the logarithmic accuracy over the scattering (but not over an external field). The matrix elements entering into the mass correction have in the used approximation the following form

$$
\begin{aligned}
& M_{1}=\left\langle 0\left|G_{p F}^{-1}-G_{0}^{-1}\right| 0\right\rangle=\frac{1}{4 \pi} \int_{0}^{\infty} \exp (-i t)\left(\frac{\nu}{\sinh \nu t} \varphi_{p}-\frac{1}{t}\right) d t, \\
& M_{2}=\left\langle 0\left|\mathbf{p}\left(G_{p F}^{-1}-G_{0}^{-1}\right) \mathbf{p}\right| 0\right\rangle=-\frac{1}{4 \pi} \int_{0}^{\infty} \exp (-i t)\left[\frac { \nu \varphi _ { p } } { \operatorname { s i n h } \nu t } \left(\frac{4 \kappa^{\prime 2}}{\nu^{2}} \tanh ^{2} \frac{\nu t}{2}\right.\right. \\
& \left.\left.+\frac{i \nu}{\sinh \nu t}\right)-\frac{i}{t^{2}}\right] d t, \\
& M_{3}=\left\langle 0\left|\left(\mathbf{e}_{-}^{*} \mathbf{p}\right)\left(G_{p F}^{-1}-G_{0}^{-1}\right)\left(\mathbf{e}_{+} \mathbf{p}\right)\right| 0\right\rangle=-\frac{\kappa^{\prime 2}}{2 \pi \nu} \int_{0}^{\infty} \exp (-i t) \frac{\varphi_{p}}{\sinh \nu t} \tanh ^{2} \frac{\nu t}{2} d t, \\
& \varphi_{p} \equiv \varphi\left(\boldsymbol{\kappa}^{\prime}, \nu, t\right)=\exp \left[-\frac{4 i \kappa^{\prime 2} t}{\nu^{2}}\left(1-\frac{2}{\nu t} \tanh \frac{\nu t}{2}\right)\right] .
\end{aligned}
$$

Integrating by parts the term containing $1 / \sinh ^{2} \nu t$ together with the subtraction term in expression for $M_{2}$ we find

$$
M_{2}=-\frac{\nu}{4 \pi} \int_{0}^{\infty} \exp (-i t) \varphi_{p} \tanh \frac{\nu t}{2}\left(\frac{4 \kappa^{\prime 2}}{\nu^{2}}+1\right) d t-M_{1}
$$

Substituting the found expressions for $M_{1}$ and $M_{2}$ into formula (2.2) and then into Eq.(2.1) we obtain the general expressions for photon masses squared under simultaneous influence of multiple scattering in a medium and an external electromagnetic field

$$
\begin{aligned}
& k_{++}^{2}=\frac{\alpha m^{2}}{2 \pi} \int_{0}^{\omega} \frac{\omega d \varepsilon}{\varepsilon \varepsilon^{\prime}} \int_{0}^{\infty} e^{-i t} \\
& \times\left[s_{2} \nu \varphi_{p} \tanh \frac{\nu t}{2}\left(\frac{4 \kappa^{\prime 2}}{\nu^{2}}+1\right)-s_{3}\left(\frac{\nu}{\sinh \nu t} \varphi_{p}-\frac{1}{t}\right)\right] d t
\end{aligned}
$$

For $k_{+-}^{2}$ we found respectively

$$
k_{+-}^{2}=-\frac{2 \alpha m^{2}}{\pi} \int_{0}^{\omega} \frac{\omega d \varepsilon}{\varepsilon \varepsilon^{\prime}} s_{3} \kappa^{\prime 2} \int_{0}^{\infty} e^{-i t} \frac{\varphi_{p}}{\nu \sinh \nu t} \tanh ^{2} \frac{\nu t}{2} d t
$$

In the absence of external field $\left(\boldsymbol{\kappa}^{\prime}=0, \varphi_{p}=1\right)$ we have

$$
\begin{aligned}
& k_{++}^{2}=\frac{\alpha m^{2}}{2 \pi} \int_{0}^{\omega} \frac{\omega d \varepsilon}{\varepsilon \varepsilon^{\prime}} \int_{0}^{\infty} e^{-i t}\left[s_{2} \nu \tanh \frac{\nu t}{2}-\frac{s_{3}}{2 t}\left(\frac{\nu}{\sinh \nu t}-\frac{1}{t}\right)\right] d t \\
& =-\frac{\alpha m^{2}}{2 \pi} \int_{0}^{\omega} \frac{\omega d \varepsilon}{\varepsilon \varepsilon^{\prime}}\left[s_{1}\left(\ln p-\psi\left(p+\frac{1}{2}\right)\right)+s_{2}\left(\psi(p)-\ln p+\frac{1}{2 p}\right)\right], \\
& k_{+-}^{2}=0
\end{aligned}
$$


where $p=i / 2 \nu, \psi(p)$ is the logarithmic derivative of the gamma function. Subsisting the result obtained into formula (1.12) we have the probability of pair creation which agrees with formula (2.10) in 8 .

In the absence of multiple scattering $(\nu \rightarrow 0)$ we get

$$
\begin{aligned}
& k_{++}^{2}=\frac{\alpha m^{2}}{\pi} \int_{0}^{\omega} \frac{\omega d \varepsilon}{\varepsilon \varepsilon^{\prime}} \int_{0}^{\infty} e^{-i t}\left[s_{2} \kappa^{\prime 2} t \varphi_{F}-\frac{s_{3}}{2 t}\left(\varphi_{F}-1\right)\right] d t, \\
& k_{+-}^{2}=-\frac{\alpha m^{2}}{2 \pi} \int_{0}^{\omega} \frac{\omega d \varepsilon}{\varepsilon \varepsilon^{\prime}} s_{3} \kappa^{\prime 2} \int_{0}^{\infty} e^{-i t} \varphi_{F} t d t
\end{aligned}
$$

where

$$
\varphi_{F}=\exp \left(-i \frac{\kappa^{\prime 2} t^{3}}{3}\right)
$$

For this case the expressions for $k_{1,2}^{2}$ (1.10) after substitution results of (2.17) agree with masses squared of photon calculated in an external electromagnetic field (see [3] and references therein).

We will analyse now the results obtained in different limiting cases. In the case when the both characteristic parameters are small $\left(\nu_{1}^{2}=4 Q L_{1} \ll 1, \kappa \ll 1\right)$, the main terms of decomposition of the functions $k^{2}$ are the sum of independent corrections to the photon mass squared both on account of the multiple scattering and an external field

$$
\begin{aligned}
& k_{++}^{2}=\frac{\alpha m^{2}}{\pi}\left[-i \frac{7}{9} \frac{\omega}{\omega_{e}}\left(1-\frac{1}{21 L_{1}}\right)+\frac{59}{225}\left(\frac{\omega}{\omega_{e}}\right)^{2}\right. \\
& \left.-i \frac{3 \sqrt{3} \pi}{16 \sqrt{2}} \frac{\omega}{\omega_{F}} \exp \left(-\frac{8 \omega_{F}}{3 \omega}\right)-\frac{11}{90}\left(\frac{\omega}{\omega_{F}}\right)^{2}\right], \\
& k_{+-}^{2}=\frac{\alpha m^{2}}{\pi}\left[i \frac{\sqrt{3} \pi}{16 \sqrt{2}} \frac{\omega}{\omega_{F}} \exp \left(-\frac{8 \omega_{F}}{3 \omega}\right)+\frac{1}{30}\left(\frac{\omega}{\omega_{F}}\right)^{2}\right] ; \quad \omega \ll \omega_{e}, \quad \omega \ll \omega_{F} .
\end{aligned}
$$

Here the notations are introduced:

$$
\omega_{e}=\frac{m}{2 \pi Z^{2} \alpha^{2} \lambda_{c}^{3} n_{a} L_{1}}, \quad \omega_{F}=m \frac{H_{0}}{|\mathbf{F}|}, \quad H_{0}=\frac{m^{2}}{e}=4.41 \cdot 10^{13} \mathrm{Oe} .
$$

For used notations see Eqs.(1.4), (2.4). The correction $\propto 1 / L_{1}$ follows from the first term of decomposition in Eq.(2.9). In gold the value $\omega_{e}$ is $\omega_{e}=10.5 \mathrm{TeV}$, this is the typical value for the heavy elements.

In Fig. the functions $\operatorname{Re} k_{++}^{2}$ (curve 2) and $\operatorname{Im} k_{++}^{2}$ (curve 1) are given for the case when the influence of a medium is taken into account only (Eq. (2.16)). The both curves are normalized to the asymptotics given by Eq. (2.19) in the limit $\omega_{F} \rightarrow \infty$.

In the case when the influence of an external field is small comparing with effect of a medium $\omega / \omega_{F} \ll\left(1+\omega / \omega_{e}\right)^{3 / 4}$ we can present the expression for $k^{2}$ as

$$
k^{2}=k^{2}\left(\omega_{c}\right)+\Delta k^{2}\left(\omega_{F}\right)
$$


Here $k^{2}\left(\omega_{c}\right)$ is the photon mass squared under influence of a medium only (in absence of an external field $\left.\kappa^{\prime}=0\right)$ is given by Eq.(2.16), where the function $L_{c}$ is defined in Eqs.(2.5) and (2.6)

$$
\nu^{2}=4 i q=i \frac{4 \varepsilon \varepsilon^{\prime}}{\omega^{2}} \frac{\omega}{\omega_{c}}, \quad \omega_{c}=\omega_{e} \frac{L_{1}}{L_{c}}, \quad L_{c} \simeq L_{1}\left[1+\frac{1}{2 L_{1}} \ln \left(1+\frac{\omega}{\omega_{e}}\right)\right] .
$$

Retaining the main terms of decomposition over $\boldsymbol{\kappa}^{\prime 2}$ in Eqs.(2.14) and (2.15) we find the corrections $\Delta k^{2}\left(\omega_{F}\right)$ :

$$
\begin{aligned}
& \Delta k_{++}^{2}\left(\omega_{F}\right)=-i \frac{\alpha m^{2}}{2 \pi} \frac{\omega \omega_{c}}{\omega_{F}^{2}} \int_{0}^{\omega} \frac{d \varepsilon}{\omega} \int_{0}^{\infty} \exp \left(-i \frac{z}{\nu}\right) \\
& \times\left\{s_{2}\left[\tanh \frac{z}{2}\left(1-i \frac{z}{\nu}\right)+\frac{2 i}{\nu} \tanh ^{2} \frac{z}{2}\right]+\frac{i s_{3}}{\nu \sinh z}\left(z-2 \tanh \frac{z}{2}\right)\right\} d z \\
& \Delta k_{+-}^{2}\left(\omega_{F}\right)=i \frac{\alpha m^{2}}{2 \pi} \frac{\omega \omega_{c}}{\omega_{F}^{2}} \int_{0}^{\omega} \frac{d \varepsilon}{\omega} s_{3} \int_{0}^{\infty} \exp \left(-i \frac{z}{\nu}\right) \frac{\tanh ^{2} \frac{z}{2}}{\sinh z} d z
\end{aligned}
$$

In the limit $\omega \gg \omega_{e}$ one can use the asymptotics found in Appendix A in 8 for calculation of the photon mass squared $k_{++}^{2}\left(\omega_{c}\right)$ :

$$
\begin{aligned}
& k_{++}^{2}\left(\omega_{c}\right)=-\frac{2 \alpha m^{2}}{\pi} \Pi(a), \quad a^{2}=i \frac{\omega_{c}}{\omega} \\
& \Pi(a) \simeq-\frac{3 \pi}{8 a}+\left(2 \ln 2-\frac{1}{2}\right) \ln \frac{1}{a}+A-\frac{\pi^{3} a}{48} \\
& A=\ln 2(2 \ln 2-1)+\frac{1}{2}(1+C)-2 \sum_{n=1}^{\infty} \frac{(-1)^{n}}{n} \ln n=0.736629
\end{aligned}
$$

It should be mentioned that in the limit $\omega_{F} \rightarrow \infty$ this formulas gives $k_{++}^{2}$ at $\omega / \omega_{e}=10$ within accuracy better than $7 \%$ and at $\omega / \omega_{e}=100$ within accuracy better than $0.3 \%$.

The corrections $\Delta k^{2}\left(\omega_{F}\right)$ due to action of external field in the same limit $\omega \gg \omega_{e}$ are

$$
\Delta k_{++}^{2}\left(\omega_{F}\right) \simeq-i \frac{2 \alpha m^{2}}{3 \pi} \frac{\omega \omega_{c}}{\omega_{F}^{2}}(1-\ln 2), \quad \Delta k_{+-}^{2}\left(\omega_{F}\right) \simeq i \frac{\alpha m^{2}}{12 \pi} \frac{\omega \omega_{c}}{\omega_{F}^{2}} .
$$

In the case when the principal effect is due to an external field, the main contribution into the photon mass squared is given by Eq.(2.17) and corrections connected with influence of multiple scattering are

$$
\begin{aligned}
& k^{2}=k^{2}\left(\omega_{F}\right)+\Delta k^{2}\left(\omega_{c}\right), \\
& \Delta k_{++}^{2}\left(\omega_{c}\right)=\frac{\alpha m^{2}}{15 \pi} \frac{\omega}{\omega_{c}} \int_{0}^{\omega} \frac{d \varepsilon}{\omega}\left\{\int_{0}^{\infty} \exp \left[-i t\left(1+\frac{1}{3} \kappa^{\prime 2} t^{2}\right)\right]\right. \\
& \left.\times\left[s_{2}\left(8 i t+3+\frac{2}{\kappa^{\prime 2}}\right)+s_{3}\left(3 i t+\frac{1}{\kappa^{\prime 2}}\right)\right] d t+\frac{i}{\kappa^{\prime 2}}\left(2 s_{2}+s_{3}\right)\right\},
\end{aligned}
$$




$$
\begin{aligned}
& \Delta k_{+-}^{2}\left(\omega_{c}\right)=-\frac{2 \alpha m^{2}}{15 \pi} \frac{\omega}{\omega_{c}} \int_{0}^{\omega} \frac{d \varepsilon}{\omega} s_{3}\left[\int_{0}^{\infty} \exp \left[-i t\left(1+\frac{1}{3} \kappa^{\prime 2} t^{2}\right)\right]\left(2 i t-3+\frac{1}{2 \kappa^{\prime 2}}\right)\right. \\
& \left.+\frac{i}{2 \kappa^{\prime 2}}\right] .
\end{aligned}
$$

Here the function $L_{c}$ is determined in Eqs. 2.5) and (2.6) at $Q=0$

$$
L_{c} \simeq L_{1}\left[1+\frac{2}{3 L_{1}} \ln \left(1+\frac{\omega}{\omega_{F}}\right)\right] .
$$

To obtain expressions Eq.(2.26) we performed decomposition in formulas (2.14) and (2.15) and integrated by parts the terms with high powers of $t$ in integrals over $t$. Note, that using Eq.(1.12) we can obtain from Eq.(2.26) the correction to the probability of pair photoproduction due to effect of weak multiple scattering. The result agrees with Eq.(7.136) in [6].

In the limiting case $\omega \gg \omega_{F}$ we have from Eq.(2.17)

$$
k_{++}^{2}\left(\omega_{F}\right) \simeq \frac{5 \alpha m^{2}}{7 \pi} e^{-i \frac{\pi}{3}} \frac{\Gamma^{3}(2 / 3)}{\Gamma(1 / 3)}\left(\frac{3 \omega}{\omega_{F}}\right)^{2 / 3}, \quad k_{+-}^{2}\left(\omega_{F}\right)=-\frac{1}{5} k_{++}^{2}\left(\omega_{F}\right) .
$$

For corrections originating from the multiple scattering we have from Eq.(2.26) the main term of decomposition

$$
\Delta k_{++}^{2}\left(\omega_{c}\right) \simeq \frac{4 \alpha m^{2}}{75 \pi} e^{-i \frac{\pi}{6}} \frac{\Gamma^{3}(1 / 3)}{\Gamma(2 / 3)}\left(\frac{3 \omega}{\omega_{c}}\right)^{1 / 3}\left(\frac{\omega_{F}}{\omega_{c}}\right)^{2 / 3}, \quad \Delta k_{+-}^{2}\left(\omega_{c}\right)=\frac{1}{2} \Delta k_{++}^{2}\left(\omega_{c}\right) .
$$

\section{Conclusion}

It is curious that in the scope of the used method (see e.g.[9]) it is possible to find many of obtained in previous section results (up to numerical coefficients) basing on very simple form of the amplitude of photon scattering

$$
M \sim \frac{\alpha}{\pi} q^{2}
$$

where $q^{2}$ is defined by the equation

$$
q^{2}=-i \frac{2 \pi \omega Z^{2} \alpha^{2} n_{a} L\left(q^{2}+m^{2}\right)}{q^{2}+m^{2}}-\frac{m^{6} \boldsymbol{\kappa}^{2}}{4\left(q^{2}+m^{2}\right)^{2}} .
$$

In the case $q_{f}^{2} \ll m^{2}\left(|q|^{2} \simeq q_{f}^{2}\right.$ ), see Eqs.(1.3) and (1.4), the imaginary part of the photon scattering amplitude in forward direction (Eqs.(3.1) and (3.2)) is defined by the value $q_{s}^{2}$ while the real part of this amplitude is a sum of the correction $\sim q_{s}^{4} / m^{2}$ and the momentum transfer due to action of a field $q_{F}^{2}$ (compare with (2.19))

$$
M \sim \frac{\alpha}{\pi} m^{2}\left[-i \frac{q_{s}^{2}(m)}{m^{2}}+\frac{q_{s}^{4}(m)}{m^{4}}-\frac{\kappa^{2}}{4}\right] .
$$


At strong multiple scattering $\left(q_{s}^{2} \gg m^{2}\right)$ and in the case $q_{F}^{2} \ll q_{s}^{2}\left(\kappa^{2} m^{6} \ll q_{s}^{6}\right)$ the value $q_{s}^{2} \simeq q_{f}^{2}$ is defined by Eqs.(1.3) and (1.4)

$$
q_{s}^{4}\left(q_{s}\right)=2 \pi \omega Z^{2} \alpha^{2} n_{a} L\left(q_{s}^{2}\right)
$$

and the photon scattering amplitude (3.1) is (compare with Eqs.(2.24) and (2.25))

$$
M \sim \frac{\alpha}{\pi}\left[e^{-i \frac{\pi}{4}} q_{s}^{2}\left(q_{s}\right)-i \frac{m^{6} \kappa^{2}}{4 q_{s}^{4}\left(q_{s}\right)}\right] .
$$

When the main effect is the action of the field $\kappa \gg m$ and in the case $q_{s}^{2}\left(q_{F}^{2}\right) \ll q_{F}^{2}\left(\kappa^{2} m^{6} \gg q_{s}^{4} q_{F}^{2}\right)$ the value $q_{F}^{2} \sim q_{f}^{2}$ we have (see (1.4))

$$
q_{F}^{6}\left(q_{F}\right)=\frac{m^{6} \kappa^{2}}{4}
$$

and the photon scattering amplitude (3.1) is (compare with Eqs. 2.28) and (2.29))

$$
M \sim \frac{\alpha}{\pi}\left[e^{-i \frac{\pi}{3}} q_{F}^{2}\left(q_{F}\right)+e^{-i \frac{\pi}{6}} q_{s}^{2}\left(q_{F}\right)\right] .
$$

It should be noted that beginning with some photon energy $\omega=\omega_{b}$ the radiative correction to the value $\operatorname{Re} \mathcal{E}_{j k}$ in the absence of a field $(\boldsymbol{\kappa}=0)$ becomes larger than $\omega_{0}^{2} / \omega^{2}$ (see (1.1)). Let us estimate $\omega_{b}$. According to formulas (3.1) and (3.2) this effect originate at values $q^{2} \ll m^{2}$. Because of this for estimate Re $M(3.1)$ it is necessary to take into account the next term in decomposition over $q^{2}$ in $(3.2)$ at $\boldsymbol{\kappa}=0$. We find

$$
\begin{aligned}
& \operatorname{Re} M_{s} \sim \frac{\alpha}{\pi} \frac{q_{s}^{4}(m)}{m^{2}}, \quad-\frac{1}{\omega} \operatorname{Im} M_{s} \sim \frac{\alpha}{\pi} \frac{q_{s}^{2}}{\omega}=\frac{1}{L_{\text {rad }}}, \\
& \frac{\operatorname{Re} M_{s}}{\omega_{0}^{2}} \sim \frac{\pi}{\alpha} \frac{\omega^{2}}{\omega_{0}^{2}} \frac{\lambda_{c}^{2}}{L_{r a d}^{2}}, \quad \omega_{b} \sim \sqrt{\frac{\alpha}{\pi}} \frac{L_{r a d}}{\lambda_{c}} \omega_{0} .
\end{aligned}
$$

For gold one obtains $\omega_{b} \sim 40 \mathrm{GeV}$.

A propagation of high-energy photons in oriented single crystals is one of interesting applications of the result obtained above. In this case we have both the dense matter with strong effect of multiple scattering and high fields of crystal axes or planes. As known, the Landau-Pomeranchuk-Migdal (LPM) effect (influence of multiple scattering on processes of bremsstrahlung and pair creation by a photon) is most pronounced in the heavy elements. The same is valid for the process under consideration. Let the high-energy photon incident on crystal. The angle of incidence is small and such that the distance from axis $\varrho$ (or the distance from plane $x$ ) can be considered as a constant on the formation length of process (the constant field approximation is applicable, see Sections 12,15 in [6]).

For orientation of a crystal along an axis the ratio of density of atoms in the vicinity of axis $n(\varrho)$ to the mean density $n_{a}$ is

$$
\xi_{a x}(\varrho)=\frac{n(\varrho)}{n_{a}}=\frac{\exp \left(-\varrho^{2} / 2 u_{1}^{2}\right)}{2 \pi u_{1}^{2} d n_{a}},
$$


where $u_{1}$ is the amplitude of thermal vibrations of atoms, $d$ is the mean distance between atoms which form the axis. This ratio is maximal at $\varrho=0$. For numerical estimates we use for definiteness the tungsten single crystal. For the axis $<111>$ in $\mathrm{W}$ the ratio $\xi_{a x}(0)=370$ at the room temperature $(T=293 \mathrm{~K})$ and $\xi_{a x}(0)=1020$ at $T=77 \mathrm{~K}$. The effect of multiple scattering becomes strong at characteristic photon energy $\omega_{e}\left(n_{a}\right) \simeq 11 \mathrm{TeV}$ and this value is inversely proportional to the density. So we have that $\omega_{e}(\varrho=0) \simeq 30 \mathrm{GeV}$ at $T=293 \mathrm{~K}$ and $\omega_{e}(\varrho=0) \simeq 11 \mathrm{GeV}$ at $T=77 \mathrm{~K}$. It should be noted that within logarithmic accuracy we neglect by relatively small variation of $L_{1}$ due to substitution the screening radius $a_{s}^{2}$ by the value $2 u_{1}^{2}$.

It is useful to compare these estimates with known threshold energies $\omega_{t}$ at which the probability of pair creation in the field of axis is equal to the probability of the Bethe-Heitler mechanism, see Table 12.1 in [6]. For photon energy $\omega \geq \omega_{t}$ the process of pair creation in the field of axis dominates. In $\mathrm{W}$ crystal for $<111>$ axis $\omega_{t}=22 \mathrm{GeV}$ at $\mathrm{T}=293 \mathrm{~K}$ and $\omega_{t}=13 \mathrm{GeV}$ at $\mathrm{T}=77 \mathrm{~K}$. It is seen that for these energies the ratio $\omega / \omega_{e}$ which characterize the strength the LPM effect is of the order of unity. At $\omega \sim \omega_{t}$ the maximal value of the parameter $\kappa(\varrho)$ which determines the probability of pair creation by a photon in a field is also of the order of unity (at $\varrho \simeq u_{1}$, see the mentioned Table). So we reach the conclusion that at some energy (for axial orientation of crystal) all the discussed effects are essential simultaneously. The analysis in this situation will be published elsewhere. For example, to calculate the influence of the field of axis on the polarization tensor one have to average the general formula (2.14) over all values of $\varrho$ (this is integration over $d^{2} \varrho$ with the weight $n_{\perp}$, where $n_{\perp}=n_{a} d$ is the density of axis in the perpendicular to them plane).

At planar orientation of crystal the ratio of the density of atoms in the vicinity of plane $n(x)$ to the mean density $n_{a}$ is

$$
\xi_{p l}(x)=\frac{n(x)}{n_{a}}=\frac{\exp \left(-x^{2} / 2 u_{1}^{2}\right) d_{p l}}{\sqrt{2 \pi} u_{1}},
$$

where $d_{p l}$ is the distance between planes. For the plane (110) in W crystal at $T=293 K$ one has $\xi_{p l}(0) \sim 18$ and effective value $\omega_{e} \simeq 600 \mathrm{GeV}$. This value is $\sim 2.5$ higher than the threshold energy $\omega_{t}$ for this plane (see Table 15.1 in [6]). Let us note that in both axial and planar cases we made estimations for the maximal value of $\xi(0)$.

In the crystals where the atomic number $Z$ is not very high $(\mathrm{Ge}, \mathrm{Si}, \mathrm{C}$ ) the ratio $\omega_{t} / \omega_{e}(0)$ is smaller than unity. So for $\omega \geq \omega_{t}$ one can use Eqs.(2.17) and (2.26) while for $\omega \ll \omega_{t}$ the formula (2.19) is applicable in which along with known results (see e.g. [6]) there is the new term in Re $k_{++}^{2}$ which is proportional to $\left(\omega / \omega_{e}\right)^{2}$.

Acknowledgements This work was supported in part by the Russian Fund of Basic Research under Grant 00-02-18007. 


\section{References}

[1] J. Schwinger, Phys.Rev. 75 (1949) 1912; 82 (1951) 664.

[2] N.B.Narozhny, Zh. Eksp.Teor.Fiz., 55 (1968) 714.

[3] V. N. Baier, V. M. Katkov, and V. S. Fadin, Radiation from Relativistic Electrons (in Russian), Atomizdat, Moscou, 1973.

[4] V. N. Baier and V. M. Katkov, Sov. Phys. JETP, 26 (1968) 854;28 (1969) 807.

[5] V. B. Berestetskii, E. M. Lifshitz, and L. P. Pitaevskii, Quantum Electrodynamics, 2nd ed. Pergamon Press, Oxford, 1982.

[6] V.N.Baier, V.M.Katkov and V.M.Strakhovenko, Electromagnetic Processes at High Energies in Oriented Single Crystals, World Scientific Publishing Co, Singapore, 1998.

[7] V. N. Baier and V. M. Katkov, Phys.Rev. D 57 (1998) 3146, hep-ph/9709214.

[8] V. N. Baier and V. M. Katkov, Phys.Rev.D 62 (2000) 036008, hep-ph/0002097.

[9] V.N.Baier, V.M.Katkov, Phys.Rev.D 63 (2001) 116008, hep-ph/0005215.

[10] V.N.Baier, V.M.Katkov, Phys.Lett.A 280 (2001) 275.

hep-ph/0011340, 


\section{Figure caption}

The functions Re $k_{++}^{2}$ (curve 2) and $\operatorname{Im} k_{++}^{2}$ (curve 1) versus the photon energy taken in units $\omega_{e}$ (because of this the curves are universal) for the case when the influence of a medium is taken into account only (Eq.(2.16)). The both curves are normalized to the asymptotics given by Eq.(2.19) in the limit $\omega_{F} \rightarrow \infty$. 